\title{
A young man presenting with pleural effusion: presumably due to acute pancreatitis from sodium valproate
}

\author{
MCB Galahitiyawa ${ }^{1}$, JDVC Lekamwasam ${ }^{2}$, RMSK Ratnayake ${ }^{3}$, P Manivannan ${ }^{1}$, MRP \\ Weerawansa $^{1}$ \\ ${ }^{1}$ Registrar in Medicine, ${ }^{2}$ Consultant Physician, ${ }^{3}$ Senior Registrar in Medicine, \\ Karapitiya Teaching Hospital, Galle.
}

\begin{abstract}
A 24 year old patient with seizure disorder and mental subnormality since birth, presented with left hypochondrial pain, shortness of breath, cough and left sided pleuritic type chest pain of two weeks duration. He had no fever, but was anorexic during this illness. Patient had been taking phenobarbitone $60 \mathrm{mg}$ bid and phenytoin sodium $100 \mathrm{mg}$ bid for 23 years and sodium valproate $400 \mathrm{mg}$ bid had been added to achieve a better control of seizers 2 months ago. The last convulsion was 2 months ago and there was no history of recent fall or trauma to the chest.
\end{abstract}

On admission patient was afebrile, dyspnoeic, and emaciated. There was a left sided pleural effusion involving the lower and middle zones while the right hypochondrium was mildly tender. Except for ESR of $40 \mathrm{~mm}$, initial investigations including full blood count, blood picture, urinalysis, blood urea, serum creatinine and clotting profile were normal. Sputum was negative for AFB and malignant cells while Mantoux test was negative. Pleural aspiration was uniformly blood stained and the possibility of acute pancreatitis was considered. CT of the abdomen showed enlarged and oedematous pancreas. His serum amylase was found to be high which confirmed the diagnosis of acute pancreatitis. Pleural fluid amylase was also found to be high.

He was managed conservatively with intravenous fluid and antibiotics but the general condition of the patient continued to deteriorate and daily pleural fluid drain remained undiminished. The possibility of drug induced acute pancreatitis was considered and sodium valproate was withdrawn from his drug regime. The patient made a dramatic recovery within few days and the pleural aspirate ceased.
Although rare, acute pancreatitis has been reported with the long term use of sodium valproate $[1,2]$. The clinical features of acute pancreatitis were subtle in our patient and major attention was paid to the moderately large pleural effusion. Blood stained pleural effusion always raise the possibility of either primary or secondary malignant diseases. However among other well recognised causes of blood stained effusion such as uremia and recent trauma, the possibility of acute pancreatitis should always be considered. When this occurs, one would expect clinical features of acute pancreatitis to be obvious but subtle clinical features made the diagnosis difficult in our patient. One may argue that the incrimination of sodium valproate as the aetiological cause of effusion is not justifiable without reintroducing the drug to see the recurrence of the effusion. This was not done as we considered it to be unethical.

\section{References}

1. David A Warrell, Timothy M Cox, John D Firth, Edward J Benz Jr. Oxford Textbook of Medicine. Oxford University Press. $4^{\text {th }}$ edition. 2003; 2: 680.

2. Dennis L Kasper, Engene Braunwald, Anthony S Fauci, Stephen L Hanser, Dan L Longo, J Larry Jameson. Harrison's Principles of Internal Medicine. McGraw Hill Medical Publication Division. $16^{\text {th }}$ edition, page 1896. 